\title{
Cancer-Related Chronic Pain
}

\author{
Examining Quality of Life in Diverse Cancer Survivors
}

Carmen R. Green, MD ${ }^{1,2,3}$; Tamera Hart-Johnson, $\mathrm{MS}^{1}$; and Deena R. Loeffler, $\mathrm{MA}^{1}$

BACKGROUND: Disparities in cancer survival and pain rates negatively impact quality of life (QOL). This study examines cancer-related chronic pain (CP) and its impact on QOL in diverse cancer survivors. METHODS: This survey study focused on current and past pain, health, and QOL in black and white cancer survivors. Participants with breast, colorectal, lung, and prostate cancer and multiple myeloma were recruited through the Michigan State Cancer Registry. Analysis of variance was used to examine outcome differences by pain status, race, and sex. Hierarchical regressions explored predictors for experiencing pain. RESULTS: The subjects ( $N=199)$ were $31 \%$ black, $49 \%$ female, and 57 to 79 years old; $19.5 \%$ experienced current pain, and $42.6 \%$ reported pain since diagnosis. Women experience more pain $(P<.001)$ and greater pain severity $(P=.04)$ than men. Blacks experienced more pain interference and disability $(P<.05)$. Experiencing pain is related to greater depressive symptoms, poorer functioning, and more symptoms. In hierarchical regressions, female sex predicted pain since diagnosis; pain severity for pain since diagnosis was predicted by black race and female sex. CONCLUSIONS: The authors extend the literature by showing that $20 \%$ of diverse cancer survivors had cancer-related CP, and $43 \%$ had experienced pain since diagnosis, revealing racial and sex disparities in cancer-related CP's incidence and impact on QOL. Having pain was related to poorer QOL in several domains and was more frequently experienced by women. Although black race was not related to pain prevalence, it was related to greater severity. This study reveals an unaddressed cancer survivorship research, clinical, and policy issue. Cancer 2011;117:1994-2003. () 2010 American Cancer Society.

KEYWORDS: cancer, pain, quality of life, health, survivorship, sex, race and ethnicity, disparities, cancer-related chronic pain.

Chronic pain, the third largest global public health problem, remains a neglected topic in medicine. ${ }^{1-5}$ Pain complaints and cancer are increasing as more people survive cancer and chronic diseases. ${ }^{5,6}$ In the US, pain is the most frequent cause of disability and second leading cause for physician visits, and is associated with depression, anxiety, and sleep disturbance. ${ }^{7,8}$ The National Cancer Institute (NCI) estimates most $(>60 \%)$ people diagnosed with cancer will be alive in 5 years. ${ }^{9}$ According to the Lance Armstrong Foundation and NCI, survivorship begins at diagnosis; however, pain threatens health and quality of life (QOL). ${ }^{10}$ Most (60\%-85\%) people with advanced cancer and nearly $40 \%$ of 5 -year survivors report pain. ${ }^{1,11-13}$ Many unscheduled hospital admissions (14\%-26\%) are because of uncontrolled pain, costing $>\$ 10$ million in expenditures. ${ }^{14}$ However, cancer-related chronic pain (CP) remains a poorly explored survivorship issue, especially among diverse cancer survivors. ${ }^{6,15-18}$ As America ages and diversifies the increasing prevalence of cancer, pain, and cancer-related CP will have significant individual and societal ramifications. ${ }^{19}$

Increased longevity and QOL have not been uniformly translated. ${ }^{20}$ Minorities are more likely to report diminished health, less likely to have a physician, have less access to specialists, and experience increased disease burden, even when their health insurance and socioeconomic status are similar to whites. ${ }^{1,21-25}$ Cancer remains the second leading cause of death in the United States, and significant disparities exist. ${ }^{18,24,26}$ Despite increased awareness, a knowledge gap exists regarding cancer-related $\mathrm{CP}$ in diverse cancer survivors.

The literature documents the benefits of optimizing pain management, but disturbing variability and disparities based on sociodemographic characteristics remain. ${ }^{1-4,12,18}$ Bernabei revealed black nursing home residents with cancer pain were $63 \%$ more likely than whites to receive no pain medications. ${ }^{27}$ Sex-based differences in pain care are known, with women referred to pain specialists less frequently than men. ${ }^{2-4}$ Also, structural barriers prevent minorities from being able to fill their

Corresponding author: Carmen R. Green, MD, University of Michigan, Department of Anesthesiology, $1 \mathrm{H} 247$ University Hospital, 1500 East Medical Center Drive SPC 5048, Ann Arbor, MI 48109-5048; Fax: (734) 936-9091; carmeng@med.umich.edu

${ }^{1}$ Department of Anesthesiology, University of Michigan, Ann Arbor, Michigan; ${ }^{2}$ Department of Obstetrics and Gynecology, University of Michigan, Ann Arbor, Michigan; ${ }^{3}$ Department of Health Management and Policy, University of Michigan, Ann Arbor, Michigan

DOI: 10.1002/cncr.25761, Received: June 2, 2010; Revised: September 24, 2010; Accepted: October 4, 2010, Published online November 18, 2010 in Wiley Online Library (wileyonlinelibrary.com) 
prescriptions in their local pharmacies. ${ }^{28,29}$ Critically important data to ensure appropriate pain and healthcare planning are lacking, yielding a significant literature gap. ${ }^{18}$

Despite the importance of cancer, pain, and health disparities, it is unknown whether the increased health perturbations seen with $\mathrm{CP}$ are more pronounced in cancer survivors. This study in black and white survivors hypothesizes that the prevalence, etiology, characteristics, and sequelae (eg, QOL) of cancer-related CP vary by race and sex. This study was designed to 1) identify and compare the relative prevalence, etiology, and characteristics of cancer-related $\mathrm{CP} ; 2)$ investigate and compare the relationship between cancer-related $\mathrm{CP}$ and mental health, disability, and QOL; and 3) evaluate and compare sociodemographics, attitudes, and pain care factors influencing cancer-related $\mathrm{CP}$ potentially mediating the relations described above.

\section{MATERIALS AND METHODS}

\section{Participants}

The University of Michigan Health System Institutional Review Board and the Michigan State Cancer Registry Institutional Review Board granted study approval. Each participant provided written informed consent upon enrollment. Adult survivors (18-90 years old) who experienced breast, prostate, colorectal, or lung cancer or multiple myeloma with initial diagnosis at least 2 years prior provided study data. Survivor was defined in accordance with the Lance Armstrong Foundation and NCI, which define survival from the moment of diagnosis. Because of geographic reporting inconsistencies, we used diagnosis plus 2 years to ensure consistency. ${ }^{10}$

The Michigan State Cancer Registry stratified their database by race and randomly identified black and nonHispanic white potential subjects, cross-checked death records, verified addresses, and contacted physicians to ensure there were not contraindications to participation. Up to 3 letters were mailed, the last certified, with an interest form and return envelope. Information from participants returning signed interest forms was forwarded from Michigan State Cancer Registry to the study team. The research office then mailed participants' information and the survey using Dillman's Total Design Method. ${ }^{30}$ A modest $\$ 20$ incentive was sent on receipt of the survey.

\section{Measures}

Sociodemographics included age, self-identified race, sex, marital status, education, employment, and household income.
Cancer and treatment information was self-reported and confirmed through registry report. Cancer information included diagnosis, date, stage, and current status (cancer-free, remission, active and receiving treatment, active and not receiving treatment). A treatment checklist was included: chemotherapy, radiation therapy, hormone therapy, and surgery. Participants could check all that applied and marked when they had last received each treatment (never, 0-3 months ago, 3-12 months ago, 1-2 years ago, $>2$ years ago, do not recall).

Current pain was used to establish skip patterns in the survey, with the question, "Do you currently experience pain regularly?" Participants were asked to classify pain: consistent pain, consistent pain that sometimes had much stronger pain flares, pain flares only, or pain only related to certain activities or motions. The same screening questions were again asked related to pain since cancer.

The Brief Pain Inventory (BPI) assessed pain severity and interference with normal functioning. ${ }^{31}$ Items determined pain severity (at worst, least, average, and right now), pain-related interference (eg, mood, work, relationships, sleep), pain characteristics (eg, timing, duration, quality), pain location (via 2 drawings of the body), pain duration and description (ie, consistent, flares, or both), pain-related medications and percentage of pain relieved by medication, and an open-ended item regarding what actions relieved pain. A subset of questions was repeated for past pain, in addition to documenting the timing of cancer pain when the participant was no longer experiencing pain.

The Center for Epidemiological Studies Depression Scale assessed depressed affect. We dropped the 4 positively worded items from the original 20-item survey, because factor analysis showed the items do not accurately predict negative affect when reversed, as confirmed in the literature for cancer patients. ${ }^{32}$ Responses for the remaining 16 items were summed and weighted to calculate an overall score comparable to the published scale range (0-60; scores $>15$ indicated severe psychological distress and depression). Internal consistency was consistent with published values $(\alpha=.91){ }^{32}$

The John Henryism Active Coping Scale evaluated a high output active coping strategy characterized by protracted struggles against seemingly insurmountable obstacles. Originally reported in older blacks, it is correlated with high blood pressure and bodily pain. The sum score of 12 Likert-type items was calculated $(60=$ maximum active coping score). Internal consistency ( $\alpha=.87)$ was higher than published values. ${ }^{33}$ 
The Barriers Questionnaire assessed attitudes toward pain management and has 4 separate subscales: physiological effects, fatalism, communication, and harmful effects. ${ }^{34}$ Mean scores were calculated for each set subscale; fatalism items were reverse scored before analysis. The internal consistency for subscales ranged from $\alpha=$ .60 to $\alpha=.91$. Only the fatalism subscale had a reliability value below published values of $\alpha=.79 .{ }^{34}$ Reliability was $\alpha=.60$. It is possible that our small sample size, the few questions on the subscale, and potential differences in the meaning to survivors may account for this difference.

The Pain Disability Index (PDI) evaluated the impact of $\mathrm{CP}$ on the individual via summation of seven 11-point subscales representing 7 life domains (family, occupation, recreation, social, sexual, life-support, and self-care) and the amount of impairment $(0=$ no disability, $10=$ total disability, $70=$ maximum disability). ${ }^{35}$ PDI was weighted, allowing for up to 2 missing items.

The European Organisation for the Research and Treatment of Cancer survey assessed health-related quality of life. ${ }^{36}$ Five QOL functioning domains (eg, cognitive, emotional, physical) and 8 symptom-control domains (eg, anorexia, dyspnea, fatigue) were evaluated for their contribution to QOL. Additional measures assessed financial concerns, global health, and overall QOL. Scores were linearly transformed to a 0 to 100 scale. Internal consistency of the subscales $(\alpha=.71-.91)$ was high. ${ }^{36}$

\section{Statistical Analysis}

Descriptive statistics were calculated using PSAW 18.0. Analysis of variance and chi-square analysis were used for group comparisons; because of multiple comparisons; a conservative $P=.01$ was used to determine significance. Hierarchical logistic regression examined predictors of current pain and pain at any time since cancer diagnosis. Age and dummies for black race and female sex were entered at Block 1, income and education at Block 2. Prostate cancer, related to lower pain rates than other cancers, was used for cancer type; chemotherapy was related to current and any pain; radiation and hormone treatments were predictive of having had pain since diagnosis; likewise, cancer surgery was listed as the most common pain cause, so dummy variables were used for each treatment. All were added in the final block, followed by multivariate regression among those who had experienced pain (using the same prediction variables, to test for predictors of pain severity). Because of the smaller sample size and lack of relationship with pain severity, radiation, hormone therapy, and surgery were not included in the severity regressions.

\section{RESULTS}

\section{Participants}

Nine hundred fifty participants were identified in the 2 waves ( 450 whites, 500 blacks). Blacks were more likely to be deceased ( $\mathrm{n}=70$ vs $\mathrm{n}=33$ ), have physicians expressing contraindication ( $\mathrm{n}=45$ vs $\mathrm{n}=32$ ), and have bad addresses $(\mathrm{n}=71$ vs $\mathrm{n}=28)$; leaving 670 people $(49 \%$ female, $47 \%$ black) eligible to contact. Blacks were less likely to actively refuse participation ( $n=44$ vs $n=86$; $19.4 \%$ overall), but were also less likely to actively agree in addition to the greater mortality and mobility, so were less likely to be forwarded to the research office $(\mathrm{n}=105$ black [33.5\%]; 158 white [44.3\%]; 39.3\% overall) and represented a smaller percentage to complete surveys $(\mathrm{n}=$ 61 black [19.5\%]; 137 white [38.4\%]). Respondents $(\mathrm{N}=199)$ were $31 \%$ black, $49 \%$ female, and from 42 to 87 years old. One person reported their race as Arabic/East Asian and was not included in racial comparisons. Most participants were currently cancer free or in remission (95.2\% women, $95.7 \%$ men, $94.3 \%$ blacks, $98.2 \%$ whites). Cancer type differed by sex $(P<.001)$ but not by race. Blacks tended to have lower incomes $(P=.04)$, lower cancer stages, and lower surgery rates. Women had higher rates of chemotherapy, radiation, and hormone therapy and were more likely to lack insurance $(P=.02)$. Table 1 provides information about the sample's characteristics. Most (>90\%) of "most recent" treatments were received $>1$ year prior. Other than hormone treatment, $>85 \%$ of the other treatments had occurred $>2$ years earlier.

\section{Group Differences}

Nearly 20\% (19.5\%) experienced current pain. Women experienced more pain since diagnosis than men $(56 \%$ vs $30 \% ; P<.001$ ). Tables 2 and 3 provide data on cancer and pain characteristics by group. Pain flares were the most common pain form and were more common in women than men $(P=.03)$. Twice as many women reported more pain with activities than men $(50 \%$ vs $24 \% ; P=.05)$. The most significant source of pain was cancer surgery $(53.8 \%)$ for whites and cancer treatment (46.2\%) for blacks. Blacks with pain reported higher pain severity for pain since diagnosis $(P=.001)$. Women experienced more pain since diagnosis $(P<.001)$. Although not all results are significant, many findings trended the same direction, with blacks and women faring worse. Blacks experienced more interference from pain (mood and walking ability) and greater 
Table 1. Sociodemographic Characteristics by Group

\begin{tabular}{|c|c|c|c|c|c|c|c|}
\hline Characteristic & $\begin{array}{l}\text { Overall, } \\
N=199\end{array}$ & $\begin{array}{l}\text { Black, } \\
\mathrm{n}=61\end{array}$ & $\begin{array}{l}\text { White, } \\
n=137\end{array}$ & $\begin{array}{l}\text { Statistic } \\
(P)\end{array}$ & $\begin{array}{l}\text { Men, } \\
n=101\end{array}$ & $\begin{array}{l}\text { Women, } \\
\mathrm{n}=98\end{array}$ & $\begin{array}{l}\text { Statistic } \\
(P)\end{array}$ \\
\hline $\begin{array}{l}\text { Age, y } \\
\% \text { black } \\
\% \text { women }\end{array}$ & $\begin{array}{l}68.1 \pm 10.5 \\
31 \% \\
49 \%\end{array}$ & $\begin{array}{l}67.2 \pm 10.6 \\
- \\
47.5 \%\end{array}$ & $\begin{array}{l}68.1 \pm 10.6 \\
- \\
49.6 \%\end{array}$ & $\begin{array}{l}\mathrm{F}=0.21 \\
\chi^{2}=1.11(.57)\end{array}$ & $\begin{array}{l}71.6 \pm 7.8 \\
31.6 \% \\
-\end{array}$ & $\begin{array}{l}64.0 \pm 11.6 \\
29.6 \% \\
-\end{array}$ & $\begin{array}{l}\mathrm{F}=29.8(<.001)^{\mathrm{a}} \\
\chi 2=1.11(.57)\end{array}$ \\
\hline $\begin{array}{l}\text { Education } \\
\leq 6 \text { th grade } \\
7 \text { th- }<12 \text { th } \\
\text { High school } \\
\text { Some college } \\
\text { College graduate } \\
\text { Graduate school }\end{array}$ & $\begin{array}{l}6.4 \% \\
3.2 \% \\
26.2 \% \\
31.0 \% \\
23.0 \% \\
10.2 \%\end{array}$ & $\begin{array}{l}7.0 \% \\
5.3 \% \\
19.3 \% \\
40.4 \% \\
22.8 \% \\
5.3 \%\end{array}$ & $\begin{array}{l}6.1 \% \\
2.3 \% \\
29.0 \% \\
26.7 \% \\
22.9 \% \\
13.0 \%\end{array}$ & $\chi^{2}=11.0(.53)$ & $\begin{array}{l}8.2 \% \\
5.2 \% \\
27.8 \% \\
29.9 \% \\
17.5 \% \\
11.3 \%\end{array}$ & $\begin{array}{l}4.4 \% \\
1.1 \% \\
24.4 \% \\
32.2 \% \\
28.9 \% \\
8.9 \%\end{array}$ & $\chi^{2}=7.68(.26)$ \\
\hline $\begin{array}{l}\text { Income, \$ } \\
\quad \leq 9,999 \\
10,000-20,000 \\
20,001-40,000 \\
40,001-60,000 \\
60,001-100,000 \\
\geq 100,001\end{array}$ & $\begin{array}{l}1.7 \% \\
15.0 \% \\
26.1 \% \\
19.4 \% \\
23.3 \% \\
13.9 \%\end{array}$ & $\begin{array}{l}3.8 \% \\
28.3 \% \\
15.1 \% \\
24.5 \% \\
18.9 \% \\
9.4 \%\end{array}$ & $\begin{array}{l}0.8 \% \\
9.4 \% \\
31.5 \% \\
16.5 \% \\
26.0 \% \\
15.7 \%\end{array}$ & $\chi^{2}=24.6(.04)$ & $\begin{array}{l}2.2 \% \\
12.1 \% \\
26.4 \% \\
23.1 \% \\
23.1 \% \\
13.2 \%\end{array}$ & $\begin{array}{l}1.1 \% \\
18.2 \% \\
26.1 \% \\
9.6 \% \\
23.9 \% \\
14.8 \%\end{array}$ & $\chi^{2}=5.54(.60)$ \\
\hline No insurance & $3.2 \%$ & $3.1 \%$ & $3.5 \%$ & $\chi^{2}=0.50(.97)$ & $0 \%$ & $6.7 \%$ & $\chi^{2}=7.75(.02)$ \\
\hline $\begin{array}{l}\text { Cancer type } \\
\text { Breast } \\
\text { Colorectal } \\
\text { Lung } \\
\text { Multiple myeloma } \\
\text { Prostate } \\
\text { Other }\end{array}$ & $\begin{array}{l}40.4 \% \\
9.6 \% \\
4.8 \% \\
1.6 \% \\
41.0 \% \\
2.7 \%\end{array}$ & $\begin{array}{l}35.1 \% \\
10.5 \% \\
5.3 \% \\
3.5 \% \\
43.9 \% \\
1.8 \%\end{array}$ & $\begin{array}{l}43.2 \% \\
9.1 \% \\
4.5 \% \\
0.8 \% \\
39.4 \% \\
3.0 \%\end{array}$ & $\chi^{2}=4.56(.92)$ & $\begin{array}{l}2.1 \% \\
6.2 \% \\
5.2 \% \\
3.1 \% \\
79.4 \% \\
4.1 \%\end{array}$ & $\begin{array}{l}81.3 \% \\
13.2 \% \\
4.4 \% \\
0.0 \% \\
0.0 \% \\
1.1 \%\end{array}$ & $\chi^{2}=162.9(<.001)^{a}$ \\
\hline $\begin{array}{l}\text { Cancer stage (at worst) } \\
\text { I } \\
\text { II } \\
\text { III } \\
\text { IV } \\
\text { Unknown }\end{array}$ & $\begin{array}{l}21.9 \% \\
14.4 \% \\
7.0 \% \\
5.3 \% \\
51.3 \%\end{array}$ & $\begin{array}{l}30.8 \% \\
13.5 \% \\
5.8 \% \\
1.9 \% \\
48.1 \%\end{array}$ & $\begin{array}{l}19.5 \% \\
17.2 \% \\
7.8 \% \\
6.3 \% \\
48.1 \%\end{array}$ & $\chi^{2}=28.4(.01)^{a}$ & $\begin{array}{l}18.6 \% \\
10.3 \% \\
8.2 \% \\
5.2 \% \\
57.7 \%\end{array}$ & $\begin{array}{l}25.6 \% \\
18.9 \% \\
5.6 \% \\
5.6 \% \\
55.6 \%\end{array}$ & $\chi^{2}=8.55(.29)$ \\
\hline $\begin{array}{l}\text { Treatments received } \\
\text { Chemotherapy } \\
\text { Radiation } \\
\text { Hormone therapy } \\
\text { Surgery }\end{array}$ & $\begin{array}{l}36.2 \% \\
50.9 \% \\
11.4 \% \\
73.5 \%\end{array}$ & $\begin{array}{l}42.6 \% \\
50.0 \% \\
7.0 \% \\
58.9 \%\end{array}$ & $\begin{array}{l}33.9 \% \\
51.2 \% \\
14.0 \% \\
79.2 \%\end{array}$ & $\begin{array}{l}\chi^{2}=2.95(.23) \\
\chi^{2}=0.02(.88) \\
\chi^{2}=1.97(.37) \\
\chi^{2}=8.60(.01)^{a}\end{array}$ & $\begin{array}{l}17.2 \% \\
40.7 \% \\
5.2 \% \\
68.8 \%\end{array}$ & $\begin{array}{l}57.1 \% \\
61.9 \% \\
18.0 \% \\
78.8 \%\end{array}$ & $\begin{array}{l}\chi^{2}=30.5(<.001)^{\mathrm{a}} \\
\chi^{2}=7.89(.005)^{\mathrm{a}} \\
\chi^{2}=7.48(.006)^{\mathrm{a}} \\
\chi^{2}=2.33(.13)\end{array}$ \\
\hline
\end{tabular}

${ }^{a}$ Statistically significant.

Table 2. Pain Prevalence and Severity by Cancer Type and Stage

\section{Percentage \\ With Current \\ Pain, $n=40$}

\begin{tabular}{ll} 
Cancer type & \\
Breast, $\mathrm{n}=\mathbf{8 4}$ & $25.0 \%$ \\
Colorectal, $\mathrm{n}=17$ & $23.5 \%$ \\
Lung, $\mathrm{n}=\mathbf{9}$ & $22.2 \%$ \\
Multiple myeloma, $\mathrm{n}=3$ & $33.3 \%$ \\
Prostate, $\mathrm{n}=\mathbf{8 1}$ & $14.8 \%$ \\
Cancer stage at worst & \\
I, $\mathrm{n}=\mathbf{4 9}$ & $24.5 \%$ \\
II, $\mathrm{n}=35$ & $20.0 \%$ \\
III, $\mathrm{n}=14$ & $25.6 \%$ \\
IV, $\mathrm{n}=\mathbf{5}$ & $20.0 \%$ \\
Unknown, $\mathrm{n}=79$ & $16.5 \%$ \\
\hline
\end{tabular}

Current Pain
Severity,
$n=40$

$n=40$

$$
\begin{aligned}
& 2.47 \pm 1.71 \\
& 2.06 \pm 1.46 \\
& 4.00 \pm 1.77 \\
& 0.00^{\mathrm{a}} \\
& 1.34 \pm 1.07 \\
& \\
& 2.38 \pm 2.15 \\
& 2.39 \pm 1.17 \\
& 2.35 \pm 1.96 \\
& 1.25^{\mathrm{a}} \\
& 1.74 \pm 1.35
\end{aligned}
$$

\section{Percentage With Pain Since \\ Cancer, $\mathbf{n}=88$}

Pain Severity, $\mathrm{n}=88$

$3.49 \pm 1.93$

$4.00 \pm 2.10$

$2.92 \pm 2.04$

$7.44 \pm 2.14^{\mathrm{b}}$

$4.57 \pm 1.95$

$4.24 \pm 2.14$

$2.94 \pm 1.24$

$4.92 \pm 3.77$

$5.67 \pm 0.94$

$4.08 \pm 2.48$

${ }^{a} n=1$ in cell, there is no standard deviation.

${ }^{\mathrm{b}}$ Multiple myeloma has a significantly higher past pain level than all others. 
Table 3. Group Outcomes

\begin{tabular}{|c|c|c|c|c|c|c|c|}
\hline Outcome & $\begin{array}{l}\text { Overall, } \\
N=199\end{array}$ & $\begin{array}{l}\text { Black, } \\
\mathrm{n}=61\end{array}$ & $\begin{array}{l}\text { White, } \\
n=137\end{array}$ & $\begin{array}{l}\text { Statistic } \\
(P)\end{array}$ & $\begin{array}{l}\text { Men, } \\
n=101\end{array}$ & $\begin{array}{l}\text { Women, } \\
n=98\end{array}$ & $\begin{array}{l}\text { Statistic } \\
(P)\end{array}$ \\
\hline Currently experience pain & $20.1 \%$ & $21.3 \%$ & $19.7 \%$ & $\chi^{2}=0.32(.845)$ & $16.8 \%$ & $23.5 \%$ & $\chi^{2}=1.37(.24)$ \\
\hline Experienced pain since cancer & $44.2 \%$ & $39.3 \%$ & $46.7 \%$ & $\chi^{2}=1.73(.42)$ & $31.7 \%$ & $57.1 \%$ & $\chi^{2}=13.07(<.001)^{2}$ \\
\hline $\begin{array}{l}\text { Kind of pain (those experiencing } \\
\text { current pain) }^{\mathrm{b}}\end{array}$ & $\mathrm{n}=40$ & $n=13$ & $\mathrm{n}=27$ & & $\mathrm{n}=17$ & $n=23$ & \\
\hline Chronic consistent & $11.3 \%$ & $15.8 \%$ & $9.6 \%$ & $\chi^{2}=0.53(.47)$ & $21.7 \%$ & $6.4 \%$ & $\chi^{2}=0.27(.60)$ \\
\hline Acute consistent & $14.1 \%$ & $10.5 \%$ & $15.4 \%$ & $\chi^{2}=0.27(.60)$ & $17.4 \%$ & $12.8 \%$ & $\chi^{2}=0.09(.77)$ \\
\hline Consistent + flares & $11.3 \%$ & $15.8 \%$ & $9.6 \%$ & $\chi^{2}=0.53(.47)$ & $13.0 \%$ & $10.6 \%$ & $\chi^{2}=0.94(.33)$ \\
\hline Flares only & $43.8 \%$ & $42.1 \%$ & $44.4 \%$ & $\chi^{2}=0.03(.86)$ & $34.8 \%$ & $46.9 \%$ & $\chi^{2}=4.59(.03)$ \\
\hline Pain with activities & $41.9 \%$ & $31.5 \%$ & $45.5 \%$ & $\chi^{2}=1.12(.29)$ & $24.0 \%$ & $50.0 \%$ & $\chi^{2}=5.82(.05)$ \\
\hline Number of pain locations & $1.63 \pm 2.21$ & $0.60 \pm 1.26$ & $2.78 \pm 2.54$ & $\mathrm{~F}=5.79(.03)$ & $0.75 \pm 1.75$ & $2.27 \pm 2.37$ & $\mathrm{~F}=2.25$ \\
\hline \multicolumn{8}{|c|}{ Pain cause (multiple responses permitted) ${ }^{c}$} \\
\hline Cancer & $14.6 \%$ & $30.8 \%$ & $7.1 \%$ & $\chi^{2}=3.97(.05)$ & $12.5 \%$ & $16.0 \%$ & $\chi^{2}=0.10(.76)$ \\
\hline Cancer treatment & $32.5 \%$ & $33.3 \%$ & $32.1 \%$ & $\chi^{2}=0.01(.94)$ & $25.0 \%$ & $37.5 \%$ & $\chi^{2}=0.68(.41)$ \\
\hline Cancer surgery & $35.0 \%$ & $25.0 \%$ & $39.3 \%$ & $\chi^{2}=0.75(.39)$ & $31.3 \%$ & $37.5 \%$ & $\chi^{2}=0.17(.69)$ \\
\hline Accident/injury & $21.4 \%$ & $15.4 \%$ & $24.1 \%$ & $\chi^{2}=0.41(.52)$ & $33.3 \%$ & $12.5 \%$ & $\chi^{2}=2.65(.10)$ \\
\hline Illness/disorder & $39.0 \%$ & $30.8 \%$ & $42.9 \%$ & $\chi^{2}=0.83(.36)$ & $58.8 \%$ & $25.0 \%$ & $\chi^{2}=4.78(.03)$ \\
\hline $\begin{array}{l}\text { Percentage of pain relieved with } \\
\text { medication }(100=\text { full relief })^{c}\end{array}$ & $61.19 \%$ & $71.1 \%$ & $55.4 \%$ & $\mathrm{~F}=1.64(.21)$ & $62.6 \%$ & $60.1 \%$ & $\mathrm{~F}=0.40(.84)$ \\
\hline BPI pain severity, current pain ${ }^{c}$ & $2.07 \pm 1.61$ & $2.21 \pm 2.06$ & $2.00 \pm 1.33$ & $F=0.17(.69)$ & $1.51 \pm 1.39$ & $2.54 \pm 1.65$ & $F=4.68(.04)$ \\
\hline \multicolumn{8}{|l|}{ BPI pain interference ${ }^{c}$} \\
\hline General activity & $2.76 \pm 2.74$ & $2.86 \pm 3.25$ & $2.71 \pm 2.51$ & $\mathrm{~F}=0.25(.88)$ & $2.33 \pm 2.68$ & $3.08 \pm 2.80$ & $\mathrm{~F}=0.77(.39)$ \\
\hline Mood & $2.79 \pm 2.89$ & $4.00 \pm 3.09$ & $2.04 \pm 2.54$ & $\mathrm{~F}=4.63(.04)$ & $2.33 \pm 2.89$ & $3.19 \pm 2.91$ & $\mathrm{~F}=0.85(.36)$ \\
\hline Walking ability & $3.48 \pm 3.41$ & $4.93 \pm 3.65$ & $2.67 \pm 3.04$ & $\mathrm{~F}=4.64(.04)$ & $4.21 \pm 3.58$ & $2.87 \pm 3.21$ & $\mathrm{~F}=1.64(.21)$ \\
\hline Work & $3.76 \pm 3.24$ & $5.00 \pm 3.82$ & $3.04 \pm 2.68$ & $\mathrm{~F}=3.73(.06)$ & $3.83 \pm 3.52$ & $3.70 \pm 3.08$ & $\mathrm{~F}=0.02(.90)$ \\
\hline Relationships & $2.32 \pm 3.13$ & $2.73 \pm 3.37$ & $2.08 \pm 3.03$ & $\mathrm{~F}=0.41(.53)$ & $2.67 \pm 3.41$ & $2.04 \pm 2.95$ & $\mathrm{~F}=0.39(.53)$ \\
\hline Sleep & $3.44 \pm 3.13$ & $4.40 \pm 3.11$ & $2.93 \pm 3.07$ & $\mathrm{~F}=2.23(.14)$ & $3.63 \pm 3.42$ & $3.29 \pm 2.94$ & $F=0.12(.73)$ \\
\hline Enjoyment of life & $3.09 \pm 3.29$ & $4.07 \pm 3.77$ & $2.57 \pm 2.94$ & $F=2.06(.16)$ & $2.84 \pm 3.39$ & $3.29 \pm 3.28$ & $\mathrm{~F}=0.19(.66)$ \\
\hline BPI severity, pain since cancer & $3.94 \pm 2.12$ & $5.35 \pm 1.87$ & $3.35 \pm 1.96$ & $\mathrm{~F}=13.18(.001)^{\mathrm{a}}$ & $5.03 \pm 2.06$ & $3.44 \pm 1.97$ & $\mathrm{~F}=8.13(.006)^{\mathrm{a}}$ \\
\hline \multicolumn{8}{|l|}{ Attitudes about pain treatment } \\
\hline Fatalism & $2.30 \pm 0.92$ & $2.58 \pm 0.97$ & $2.18 \pm 0.87$ & $\mathrm{~F}=7.32(.008)^{\mathrm{a}}$ & $2.28 \pm 0.91$ & $2.33 \pm 0.93$ & $F=0.11(.74)$ \\
\hline Physical barriers & $2.76 \pm 0.82$ & $2.80 \pm 0.92$ & $2.75 \pm 0.78$ & $\mathrm{~F}=0.15(.70)$ & $2.88 \pm 0.80$ & $2.67 \pm 0.84$ & $\mathrm{~F}=2.90(.09)$ \\
\hline Communication barriers & $1.96 \pm 0.84$ & $1.89 \pm 0.82$ & $1.99 \pm 0.85$ & $\mathrm{~F}=0.48(.49)$ & $1.97 \pm 0.78$ & $1.97 \pm 0.90$ & $\mathrm{~F}=0.00(.97)$ \\
\hline Harmful effects & $2.94 \pm 1.03$ & $3.18 \pm 1.17$ & $2.83 \pm 0.94$ & $\mathrm{~F}=4.19(.04)$ & $3.11 \pm 0.99$ & $2.82 \pm 1.05$ & $\mathrm{~F}=3.44(.07)$ \\
\hline \multicolumn{8}{|l|}{ Pain Disability Index } \\
\hline Family & $3.65 \pm 3.42$ & $5.50 \pm 3.70$ & $2.76 \pm 2.95$ & $\mathrm{~F}=6.91(.01)^{\mathrm{a}}$ & $3.56 \pm 3.38$ & $3.72 \pm 3.52$ & $\mathrm{~F}=0.02(.88)$ \\
\hline Recreation & $4.23 \pm 3.35$ & $5.80 \pm 3.59$ & $3.41 \pm 2.96$ & $\mathrm{~F}=5.56(.02)$ & $4.79 \pm 3.29$ & $3.80 \pm 3.39$ & $\mathrm{~F}=0.94(.34)$ \\
\hline Social activity & $3.02 \pm 3.41$ & $4.00 \pm 3.91$ & $2.52 \pm 3.08$ & $\mathrm{~F}=1.91(.18)$ & $3.74 \pm 3.54$ & $2.48 \pm 3.28$ & $\mathrm{~F}=1.48(.23)$ \\
\hline Occupation & $3.49 \pm 3.87$ & $5.21 \pm 4.40$ & $2.66 \pm 3.35$ & $\mathrm{~F}=4.47(.04)$ & $3.26 \pm 3.89$ & $3.67 \pm 3.93$ & $\mathrm{~F}=0.11(.74)$ \\
\hline Sexual behavior & $4.77 \pm 4.40$ & $5.93 \pm 4.28$ & $4.14 \pm 4.41$ & $\mathrm{~F}=1.64(.21)$ & $5.79 \pm 4.44$ & $3.96 \pm 4.29$ & $\mathrm{~F}=1.87(.18)$ \\
\hline Self-care & $1.82 \pm 3.28$ & $4.33 \pm 4.45$ & $0.52 \pm 1.21$ & $\mathrm{~F}=19.0(<.001)^{\mathrm{a}}$ & $1.63 \pm 3.34$ & $1.96 \pm 3.30$ & $\mathrm{~F}=0.11(.75)$ \\
\hline Life support & $1.73 \pm 2.80$ & $3.47 \pm 3.56$ & $0.83 \pm 1.79$ & $\mathrm{~F}=10.8(.002)^{\mathrm{a}}$ & $2.05 \pm 3.17$ & $1.48 \pm 2.52$ & $\mathrm{~F}=0.45(.51)$ \\
\hline Depression & $10.5 \pm 9.2$ & $11.5 \pm 10.6$ & $9.8 \pm 8.4$ & $\mathrm{~F}=1.47(.23)$ & $8.6 \pm 7.2$ & $12.4 \pm 10.6$ & $\mathrm{~F}=7.78(.006)^{\mathrm{a}}$ \\
\hline \multicolumn{8}{|l|}{ Functioning } \\
\hline General health & $78.3 \pm 17.4$ & $73.8 \pm 19.8$ & $80.2 \pm 16.0$ & $\mathrm{~F}=5.25(.02)$ & $79.6 \pm 16.2$ & $76.7 \pm 18.8$ & $\mathrm{~F}=1.26(.26)$ \\
\hline Physical & $82.9 \pm 20.9$ & $75.8 \pm 25.7$ & $86.0 \pm 17.6$ & $\mathrm{~F}=9.70(.002)^{\mathrm{a}}$ & $85.0 \pm 20.6$ & $80.2 \pm 21.0$ & $\mathrm{~F}=2.50(.12)$ \\
\hline Role & $86.5 \pm 24.9$ & $83.3 \pm 31.8$ & $87.9 \pm 21.2$ & $\mathrm{~F}=1.33(.25)$ & $89.2 \pm 21.9$ & $83.2 \pm 27.6$ & $F=2.76(.10)$ \\
\hline Emotional & $79.5 \pm 20.9$ & $78.1 \pm 23.1$ & $80.0 \pm 19.9$ & $\mathrm{~F}=0.33(.57)$ & $81.8 \pm 18.9$ & $76.6 \pm 22.5$ & $\mathrm{~F}=3.01(.08)$ \\
\hline Cognitive & $82.8 \pm 24.5$ & $80.7 \pm 25.7$ & $83.7 \pm 24.0$ & $F=0.60(.44)$ & $86.1 \pm 18.7$ & $78.8 \pm 29.1$ & $\mathrm{~F}=4.28(.04)$ \\
\hline Social & $87.3 \pm 23.3$ & $81.3 \pm 31.5$ & $89.8 \pm 18.3$ & $F=5.43(.02)$ & $87.0 \pm 22.7$ & $87.0 \pm 24.2$ & $F=0.00(1.0)$ \\
\hline \multicolumn{8}{|l|}{ Symptoms } \\
\hline Fatigue & $24.2 \pm 24.8$ & $27.4 \pm 30.2$ & $22.8 \pm 22.1$ & $F=1.28(.26)$ & $21.1 \pm 24.1$ & $28.2 \pm 25.2$ & $F=3.83(.05)$ \\
\hline Nausea/vomiting & $5.2 \pm 17.0$ & $8.4 \pm 18.9$ & $3.8 \pm 15.9$ & $\mathrm{~F}=3.11(.08)$ & $4.1 \pm 12.7$ & $6.8 \pm 20.8$ & $\mathrm{~F}=1.16(.28)$ \\
\hline Pain & $19.5 \pm 25.5$ & $20.3 \pm 29.0$ & $19.2 \pm 24.1$ & $\mathrm{~F}=0.07(.79)$ & $16.8 \pm 23.4$ & $23.0 \pm 27.4$ & $\mathrm{~F}=2.68(.10)$ \\
\hline Trouble sleeping & $26.0 \pm 31.3$ & $27.9 \pm 36.7$ & $25.3 \pm 28.9$ & $\mathrm{~F}=0.27(.60)$ & $24.7 \pm 30.5$ & $28.1 \pm 32.5$ & $\mathrm{~F}=0.52(.47)$ \\
\hline Appetite & $8.3 \pm 19.91$ & $13.5 \pm 24.3$ & $6.1 \pm 17.4$ & $\mathrm{~F}=5.60(.02)$ & $6.2 \pm 15.5$ & $11.0 \pm 23.9$ & $\mathrm{~F}=2.72(.10)$ \\
\hline Shortness of breath & $16.2 \pm 26.1$ & $19.3 \pm 28.1$ & $14.9 \pm 25.2$ & $\mathrm{~F}=1.13(.29)$ & $14.1 \pm 23.0$ & $19.0 \pm 29.0$ & $\mathrm{~F}=1.69(.19)$ \\
\hline Constipation & $14.7 \pm 27.4$ & $24.6 \pm 32.4$ & $10.4 \pm 23.8$ & $\mathrm{~F}=11.1(.001)^{\mathrm{a}}$ & $14.1 \pm 25.4$ & $15.9 \pm 29.7$ & $\mathrm{~F}=0.21(.65)$ \\
\hline Diarrhea & $8.2 \pm 20.2$ & $11.7 \pm 24.8$ & $6.6 \pm 17.7$ & $\mathrm{~F}=2.53(.11)$ & $6.5 \pm 15.7$ & $15.0 \pm 27.5$ & $\mathrm{~F}=1.67(.20)$ \\
\hline Financial difficulty & $13.8 \pm 28.2$ & $21.4 \pm 35.1$ & $10.6 \pm 24.1$ & $\mathrm{~F}=5.95(.02)$ & $9.7 \pm 21.6$ & $18.7 \pm 33.4$ & $\mathrm{~F}=4.79(.03)$ \\
\hline
\end{tabular}

BPI indicates Brief Pain Inventory.

a Statistically significant.

${ }^{\mathrm{b}}$ Chronic, $>3$ months; acute, $<3$ months; consistent means steady pain; flares are pain that varies greatly in strength, coming and going

${ }^{c}$ Question were asked only of those with current cancer-related pain. 
Table 4. Outcomes by Pain Status

\begin{tabular}{|c|c|c|c|c|c|c|}
\hline Outcome & $\begin{array}{l}\text { Current Pain, } \\
n=40\end{array}$ & $\begin{array}{l}\text { No Current } \\
\text { Pain, } \\
n=159\end{array}$ & $\begin{array}{l}\text { Statistic } \\
(P)\end{array}$ & $\begin{array}{l}\text { Pain Since } \\
\text { Diagnosis, } \\
n=88\end{array}$ & $\begin{array}{l}\text { No Pain Since } \\
\text { Diagnosis, } \\
n=111\end{array}$ & $\begin{array}{l}\text { Statistic } \\
(P)\end{array}$ \\
\hline \multicolumn{7}{|l|}{ Pain treatment attitudes } \\
\hline Fatalism & $2.51 \pm 0.97$ & $2.26 \pm 0.89$ & $F=2.21(.14)$ & $2.28 \pm 0.96$ & $2.33 \pm 0.88$ & $\mathrm{~F}=0.11(.74)$ \\
\hline Physical barriers & $2.87 \pm 0.78$ & $2.74 \pm 0.83$ & $\mathrm{~F}=0.73(.40)$ & $2.71 \pm 0.80$ & $2.81 \pm 0.84$ & $\mathrm{~F}=0.66(.42)$ \\
\hline Communication barriers & $2.05 \pm 0.85$ & $1.94 \pm 0.84$ & $\mathrm{~F}=0.50(.48)$ & $1.92 \pm 0.84$ & $1.99 \pm 0.85$ & $F=0.22(.64)$ \\
\hline Harmful effects & $3.06 \pm 1.03$ & $2.93 \pm 1.03$ & $\mathrm{~F}=0.47(.49)$ & $2.74 \pm 0.98$ & $3.12 \pm 1.04$ & $\mathrm{~F}=6.11(.01)^{\mathrm{a}}$ \\
\hline $\begin{array}{l}\text { Depression } \\
\text { Functioning }\end{array}$ & $13.8 \pm 11.1$ & $9.6 \pm 8.5$ & $\mathrm{~F}=6.12(.02)$ & $12.4 \pm 11.0$ & $8.9 \pm 7.3$ & $\mathrm{~F}=6.63(.01)^{\mathrm{a}}$ \\
\hline General health & $69.7 \pm 18.8$ & $80.3 \pm 16.4$ & $\mathrm{~F}=11.6(.001)^{\mathrm{a}}$ & $74.4 \pm 18.7$ & $80.9 \pm 15.9$ & $\mathrm{~F}=6.50(.01)^{\mathrm{a}}$ \\
\hline Physical & $68.5 \pm 26.5$ & $86.4 \pm 17.4$ & $\mathrm{~F}=25.1(<.001)^{\mathrm{a}}$ & $77.0 \pm 25.1$ & $87.4 \pm 15.4$ & $\mathrm{~F}=12.02(.001)^{\mathrm{a}}$ \\
\hline Role & $68.0 \pm 35.0$ & $90.9 \pm 19.3$ & $\mathrm{~F}=29.1(<.001)^{\mathrm{a}}$ & $77.6 \pm 31.3$ & $93.0 \pm 15.9$ & $\mathrm{~F}=19.9(<.001)^{\mathrm{a}}$ \\
\hline Emotional & $74.1 \pm 23.6$ & $80.8 \pm 20.0$ & $\mathrm{~F}=3.05(.08)$ & $74.2 \pm 23.1$ & $83.4 \pm 18.1$ & $\mathrm{~F}=9.51(.002)^{\mathrm{a}}$ \\
\hline Cognitive & $77.0 \pm 26.2$ & $84.1 \pm 23.8$ & $\mathrm{~F}=2.52(.11)$ & $76.5 \pm 29.3$ & $87.3 \pm 18.8$ & $\mathrm{~F}=9.46(.002)^{\mathrm{a}}$ \\
\hline Social & $90.3 \pm 20.1$ & $90.3 \pm 20.1$ & $\mathrm{~F}=15.3(<.001)^{\mathrm{a}}$ & $81.0 \pm 26.4$ & $91.7 \pm 19.6$ & $\mathrm{~F}=10.26(.002)^{\mathrm{a}}$ \\
\hline \multicolumn{7}{|l|}{ Symptoms } \\
\hline Fatigue & $45.4 \pm 29.7$ & $19.1 \pm 20.5$ & $\mathrm{~F}=38.5(<.001)^{\mathrm{a}}$ & $35.6 \pm 27.2$ & $15.7 \pm 18.7$ & $\mathrm{~F}=34.5(<.001)^{\mathrm{a}}$ \\
\hline Nausea/vomiting & $5.6 \pm 13.0$ & $5.2 \pm 17.9$ & $\mathrm{~F}=0.02(.90)$ & $3.4 \pm 9.7$ & $6.7 \pm 20.8$ & $\mathrm{~F}=1.79(.18)$ \\
\hline Pain & $45.4 \pm 31.3$ & $13.6 \pm 19.5$ & $\mathrm{~F}=59.6(<.001)^{\mathrm{a}}$ & $30.2 \pm 28.9$ & $11.9 \pm 19.2$ & $\mathrm{~F}=27.2(<.001)^{\mathrm{a}}$ \\
\hline Trouble sleeping & $41.7 \pm 38.5$ & $22.9 \pm 28.5$ & $\mathrm{~F}=11.0(.001)^{\mathrm{a}}$ & $36.3 \pm 33.2$ & $19.3 \pm 28.1$ & $\mathrm{~F}=14.5(<.001)^{\mathrm{a}}$ \\
\hline Appetite & $16.2 \pm 25.6$ & $6.5 \pm 17.9$ & $\mathrm{~F}=7.34(.007)^{\mathrm{a}}$ & $12.3 \pm 22.6$ & $5.5 \pm 17.2$ & $\mathrm{~F}=5.71(.02)$ \\
\hline Shortness of breath & $25.2 \pm 32.8$ & $14.1 \pm 23.7$ & $\mathrm{~F}=5.62(.02)$ & $20.2 \pm 29.2$ & $13.3 \pm 23.1$ & $\mathrm{~F}=3.26(.07)$ \\
\hline Constipation & $25.2 \pm 31.8$ & $12.2 \pm 25.6$ & $\mathrm{~F}=6.99(.009)^{\mathrm{a}}$ & $18.8 \pm 29.0$ & $11.8 \pm 25.8$ & $F=3.02(.08)$ \\
\hline Diarrhea & $11.7 \pm 19.6$ & $7.4 \pm 20.3$ & $\mathrm{~F}=1.36(.25)$ & $7.9 \pm 17.0$ & $8.5 \pm 22.3$ & $F=0.04(.85)$ \\
\hline Financial difficulty & $24.1 \pm 37.9$ & $11.5 \pm 24.8$ & $F=6.03(.02)$ & $20.8 \pm 34.1$ & $8.8 \pm 21.5$ & $\mathrm{~F}=8.88(.003)$ \\
\hline
\end{tabular}

a Statistically significant.

pain-related disability in several PDI domains, expressed more fatalism and concerns about harmful pain treatment effects, had poorer general health $(P=.02)$ and physical $(P=.002)$ and social $(P=.02)$ functioning, had greater appetite symptoms $(P=.02)$, and had more constipation $(P=.001)$ than whites. Women reported poorer cognitive functioning than men $(P=.04)$ and more depressive symptoms $(P=.006)$. Both blacks and women reported more financial difficulties $(P<.05)$. Additional comparisons are in Table 3.

\section{Pain Versus No Pain}

Participants with current pain reported poorer general health $(P=.001)$ and physical $(P<.001)$, role $(P<$ $.001)$, and social $(P<.001)$ functioning. Pain since diagnosis predicts all European Organisation for the Research and Treatment of Cancer survey functioning scales $(P<$ $.01), 3$ symptom scales $(P<.01)$, and depression $(P=$ $.01)$. Participants with past pain also reported significantly greater financial difficulty $(P=.003)$. Participants experiencing pain since diagnosis endorsed pain medications' harmful effects less than those who had not experienced pain $(P=.01)$. Table 4 and Figures 1 and 2 provide additional details.

\section{Predicting Pain}

Logistic regression examined predictors for current pain and pain at any time since cancer diagnosis. Age, race, and female sex were entered at Block 1, income and education at Block 2. Prostate cancer, chemotherapy, radiation, hormone therapy, and cancer surgery were added in the final block. When logistic regression was run, there were no significant predictors for current pain. Female sex predicted having had pain since the time of cancer diagnosis at block of entry. There were no significant predictors in the final model. Table 5 shows odds ratios, intervals, and significance for variables in all regressions.

Among participants with pain in multiple regression, there were no significant predictors for current pain severity as measured by BPI, but pain since diagnosis was significantly related to female sex $(\beta=-.43, P=.02)$ and black race $(\beta=.45, P<.001)$.

\section{DISCUSSION}

There are few racial differences in the prevalence of cancer by race, but stark racial disparities in morbidity and mortality. ${ }^{37}$ To our knowledge, this is the first study showing that nearly $20 \%$ of black and white cancer survivors $(>2$ 


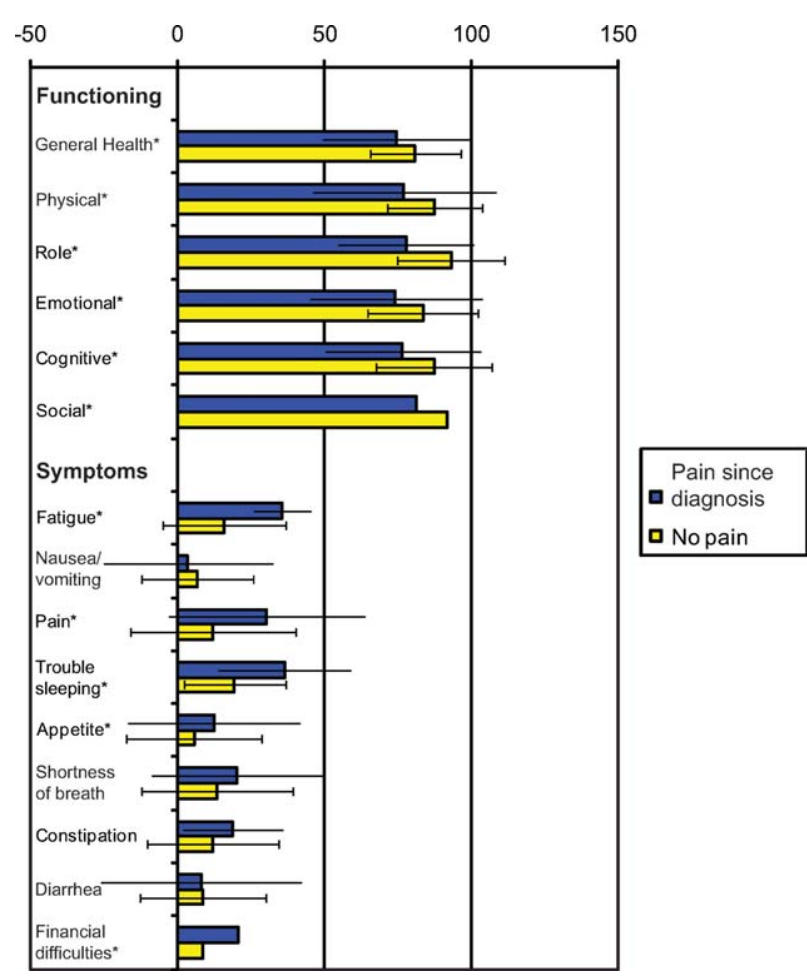

Figure 1. Current functioning and symptoms by experience of pain since diagnosis among cancer survivors are shown according to the European Organisation for the Research and Treatment of Cancer survey. All functioning and 4 symptom outcomes were significantly poorer for participants who had experienced pain.

years from diagnosis) report current chronic pain, and $44 \%$ have experienced pain since their diagnosis, comparable to previous studies. ${ }^{38,39}$ We identified differences in pain type, number of pain locations, perceived etiology, pain severity, pain interference, disability, functioning, and symptoms, with blacks and women adversely impacted. Although blacks tended to have a lower cancer stage than whites, consistent with physician variability in decision making, we identified differences in cancer treatment with women more likely to have chemotherapy and blacks having lower surgery rates. ${ }^{40}$ Although the crosssectional design cannot determine causality, our results suggest that pain associated with cancer may cause increased morbidity and diminished QOL for blacks and women, consistent with an unequal burden of cancer, pain, and cancer-related CP. ${ }^{24}$ As more people survive cancer and develop chronic pain (especially cancer-related $\mathrm{CP}$ ), these cross-cutting findings address a significant knowledge gap and survivorship issue, while highlighting significant clinical and health policy issues.

The literature reveals a significant financial toll associated with cancer. We found blacks and women reported

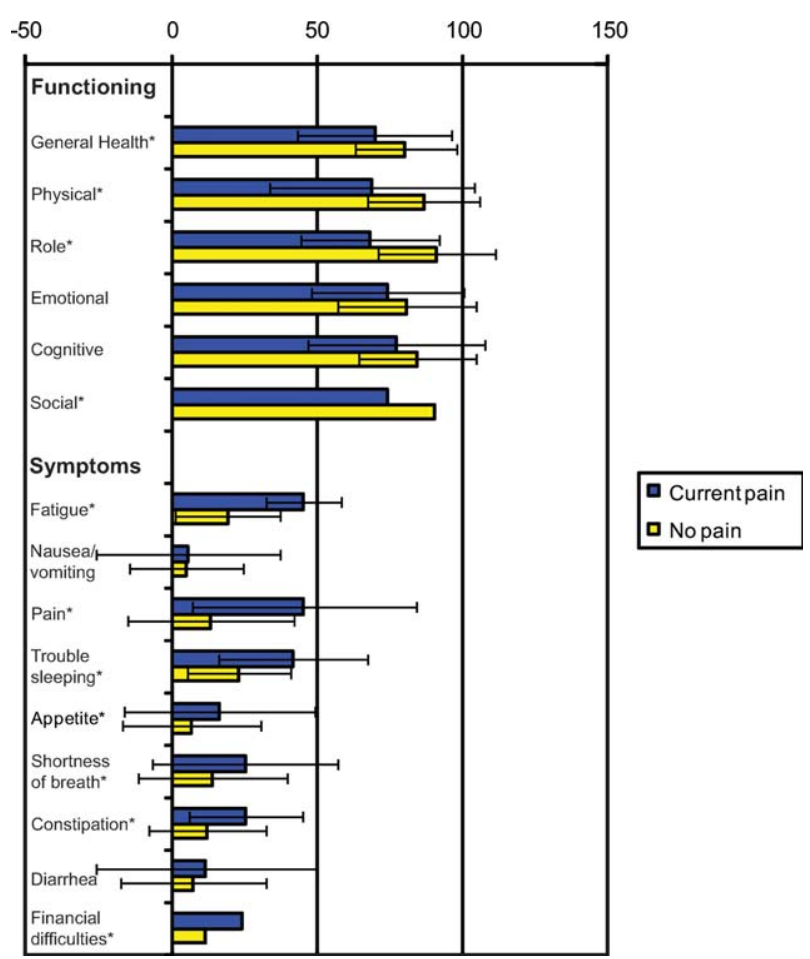

Figure 2. Current functioning and symptoms by experience of current pain among cancer survivors are shown according to the European Organisation for the Research and Treatment of Cancer survey. Current pain was related to a greater number of symptom scales, but only 4 of the 6 functioning scales.

more financial difficulties than their counterparts, with women less likely to have insurance. Blacks and women with chronic pain report increased difficulty paying for healthcare and are more likely to believe sex, race, ethnicity, and culture influence access to health and pain care. ${ }^{25,29}$ Thus, sex and race are important considerations in ensuring access to quality health and pain care. ${ }^{28,41}$ Although we did not show a difference in the prevalence of pain or depression, other investigators revealed increased pain severity and impairment for blacks. ${ }^{15,16,42,43}$ However, when blacks experienced cancer-related $\mathrm{CP}$, they had increased pain severity, depression, and disability compared with whites $(P<.05) .{ }^{18,25}$ Women had increased pain, more pain flares, more disability because of pain, and were more depressed than men $(P<.05)$. Because depression is often poorly assessed and frequently goes without treatment, they may suffer substantially more impairment in their health and QOL.

Despite significant medical advances, multimodal pain therapies, and improved longevity, most cancer survivors reporting pain reported that their pain was only relieved by $61 \%$. There are opportunities to improve the 
Table 5. Main Effect of Each Independent Variable at Block Entered and in Final Model

\begin{tabular}{|c|c|c|c|c|c|c|c|c|}
\hline Variable & $\begin{array}{l}\text { Current } \\
\text { Pain, Odds } \\
\text { Ratio (CI) }\end{array}$ & $R^{2 \mathrm{a}} / P$ & $\begin{array}{l}\text { Pain Since } \\
\text { Diagnosis, } \\
\text { Odds } \\
\text { Ratio (Cl) }\end{array}$ & $\mathrm{R}^{2 \mathrm{a}} / P$ & $\begin{array}{l}\text { Current } \\
\text { Pain, } \\
\text { BPI } \beta(P)\end{array}$ & $\begin{array}{l}\text { Current } \\
\text { Pain, } \\
\text { BPI } R^{2} \Delta\end{array}$ & $\begin{array}{l}\text { Pain Since } \\
\text { Diagnosis, } \\
\beta(P)\end{array}$ & $\begin{array}{l}\text { Pain Since } \\
\text { Diagnosis, } \\
\text { BPI } R^{2} \Delta\end{array}$ \\
\hline $\begin{array}{l}\text { Block 1: } \\
\text { demographics }\end{array}$ & & $0.025 / .41$ & & $0.148 /<.001$ & & $.123(.19)$ & & $.327(<.001)$ \\
\hline Age & $0.99(0.95-1.02)$ & 0.46 & $0.98(0.95-1.01)$ & 0.98 & $-.05(.82)$ & & $.10(.39)$ & \\
\hline Female & $1.54(0.74-2.34)$ & 0.29 & $3.08(2.42-3.74)$ & 0.001 & $.31(.13)$ & & $-.34(.005)$ & \\
\hline Black & $1.15(0.36-1.93)$ & 0.73 & $0.68(0.00-1.36)$ & 0.27 & $.09(.58)$ & & $.44(<.001)$ & \\
\hline $\begin{array}{l}\text { Block 2: } \\
\text { socioeconomic }\end{array}$ & & $0.030 / .71$ & & $0.151 / .53$ & & $.037(.48)$ & & $.020(.44)$ \\
\hline Income & $1.00(0.98-1.01)$ & 0.63 & $1.01(0.99-1.02)$ & 0.43 & $-.141(.44)$ & & $-.06(.71)$ & \\
\hline Schooling & $1.03(0.91-1.15)$ & 0.62 & $0.97(0.87-1.07)$ & 0.52 & $.18(.31)$ & & $.19(.28)$ & \\
\hline \multicolumn{9}{|l|}{$\begin{array}{l}\text { Final model } \\
\text { Demographics }\end{array}$} \\
\hline Age & $0.99(0.95-1.03)$ & 0.61 & $0.99(0.96-1.02)$ & 0.56 & $-.02(.94)$ & & $.10(.41)$ & \\
\hline Female & $0.93(0.00-2.24)$ & 0.92 & $2.05(0.94-3.16)$ & 0.21 & $.25(.38)$ & & $-.44(.02)$ & \\
\hline Black & $1.08(0.25-1.91)$ & 0.86 & $0.80(0.08-1.51)$ & 0.53 & $.14(.45)$ & & $.45(<.001)$ & \\
\hline \multicolumn{9}{|l|}{ Socioeconomic } \\
\hline Income & $1.00(0.98-1.01)$ & 0.60 & $1.01(0.99-1.02)$ & 0.44 & $-.13(.49)$ & & $-.03(.85)$ & \\
\hline Schooling & $1.04(0.84-1.24)$ & 0.69 & $0.96(0.87-1.06)$ & 0.44 & $.20(.30)$ & & $.17(.34)$ & \\
\hline $\begin{array}{l}\text { Cancer and } \\
\text { treatment }\end{array}$ & & $0.062 / .58$ & & $0.195 / .22$ & & $.013(.78)$ & & $.013(.58)$ \\
\hline Prostate cancer & $0.73(0.00-2.14)$ & 0.66 & $0.89(0.00-2.06)$ & 0.85 & $-.11(69)$ & & $-.18(.32)$ & \\
\hline Chemotherapy & $1.77(0.85-2.69)$ & 0.23 & $1.64(0.80-2.44)$ & 0.22 & $.07(.73)$ & & $-.09(.47)$ & \\
\hline Radiation therapy & $1.53(0.72-2.34)$ & 0.30 & $1.55(0.86-2.23)$ & 0.22 & & & & \\
\hline Hormone therapy & $0.72(0.00-1.17)$ & 0.58 & $1.63(0.57-2.69)$ & 0.37 & & & & \\
\hline Surgery & $1.03(0.10-1.97)$ & 0.94 & $1.82(0.80-4.43)$ & 0.15 & & & & \\
\hline
\end{tabular}

$\mathrm{Cl}$ indicates confidence interval; BPI, Brief Pain Inventory.

${ }^{a}$ Nagelkerke $R^{2}$ is included, along with significance for added block. Because logistic regression does not require normal distribution, the Nagelkerke is only an estimate.

quality of clinical care for survivors. When necessary and appropriate, both pharmacologic and nonpharmacologic therapies such as counseling should be incorporated to optimize pain care and improve QOL. However, patient and physician knowledge and attitudes about pain management (eg, opioids and nonpharmacological modalities) may contribute to suboptimal care. For instance, concerns about side effects (eg, addiction) may affect communication and coping and lead both to minimize pain complaints. ${ }^{3,4,44}$ Differences in communication styles may compromise a patient's willingness to disclose concerns and the physician's willingness to address concerns. ${ }^{45}$ For blacks and women, differences in communication styles may hamper physician decision making, but are critical for quality pain care. Future studies should design interventions to optimize physician patient communication, address attitudes regarding pain management, and decrease physician variability in decision making to improve the quality of pain care.
Having current or past pain significantly impaired health. Participants with pain reported more symptoms (including depression), decreased QOL, and increased financial difficulties. Having cancer-related CP decreased all QOL domains, with an increased health and financial burden for blacks and women. Surprisingly, there were no predictors for current pain in our final model. Pain since diagnosis was significantly related to black race and female sex. Although our findings suggest disparities based upon social determinants, future cancer-related CP studies should attempt to disentangle their role using larger diverse samples.

Despite our important findings, we acknowledge limitations. First, although the surveys had no identifiers, were collected in a confidential manner, and were chosen to determine global differences, there is potential for a self-report bias. Second, a representation bias could be present, with people in better health more willing and able to participate, and it is possible that these estimates may understate the percentage of survivors experiencing 
pain. Blacks were also more likely to be contacted to participate than whites because we needed adequate numbers for statistical analysis, thus each black had a higher probability of selection. Despite oversampling, noncoercive monetary incentives to compensate subjects, and using Dillman's Total Design Method, blacks were less likely to participate and more likely to have a bad address. This has been observed in past studies, but may also be confounded with poorer health, as blacks have earlier mortality and lower survivorship rates for cancer. ${ }^{46,47}$ Future studies should address whether these limitations are because of recruitment challenges, geographic mobility, or a survival effect. Regardless, we revealed important new findings regarding differences and disparities in cancer-related $\mathrm{CP}$, while providing a sound platform for future studies focusing on cancer survivorship in diverse samples.

In summary, we established the high prevalence (nearly 20\%) of cancer-related CP and its tremendous impact on black and white cancer survivors. More than $40 \%$ of survivors experienced pain since their cancer diagnosis, with blacks and women more impacted than whites and men. Considering the increasing prevalence of pain and cancer and persistent health disparities in an increasingly aging and diverse society, these results are particularly salient. We provide new knowledge about cancerrelated CP and its impact on overall health and QOL. We also identify important barriers, while presenting new opportunities for research focusing on interventions (eg, patient, clinician, health system, and health policy) designed to improve health and pain care quality. We provide a sound platform for research focusing on survivorship and cancer-related $\mathrm{CP}$ as well as for optimizing pain care in diverse cancer survivors. All in all, the high prevalence of cancer and pain and now cancer-related CP among diverse survivors (especially blacks and women) reveals new opportunities for clinical care, research, and policy. $^{24}$

\section{CONFLICT OF INTEREST DISCLOSURES}

We thank the Lance Armstrong Foundation, study participants, and Michigan State Cancer Registry for their generous support. The Lance Armstrong Foundation funded this study.

\section{REFERENCES}

1. Anderson KO, Richman SP, Hurley J, et al. Cancer pain management among underserved minority outpatients: perceived needs and barriers to optimal control. Cancer. 2002; 94:2295-2304
2. Green CR, Wheeler JR. Physician variability in the management of acute postoperative and cancer pain: a quantitative analysis of the Michigan experience. Pain Med. 2003;4:8-20.

3. Green CR, Wheeler JR, LaPorte F, Marchant B, Guerrero E. How well is chronic pain managed? Who does it well? Pain Med. 2002;3:56-65.

4. Green CR, Wheeler JR, LaPorte F. Clinical decision making in pain management: contributions of physician and patient characteristics to variations in practice. J Pain. 2003;4:2939.

5. Stewart WF, Ricci JA, Chee E, Morganstein D, Lipton R. Lost productive time and cost due to common pain conditions in the US workforce. JAMA. 2003;290:2443-2454.

6. Turk DC. Clinical effectiveness and cost-effectiveness of treatments for patients with chronic pain. Clin J Pain. 2002;18:355-365.

7. Greenberg PE, Leong SA, Birnbaum HG, Robinson RL. The economic burden of depression with painful symptoms. J Clin Psychiatry. 2003;64(suppl 7):17-23.

8. Green CR, Ndao-Brumblay SK, Hart-Johnson T. Sleep problems in a racially diverse chronic pain population. Clin J Pain. 2009;25:423-430.

9. Horner MJ, Ries L, Krapcho M, et al, eds. SEER Cancer Statistics Review, 1975-2006. Based on November 2008 SEER data submission, posted to the SEER web site, 2009. Bethesda, MD: National Cancer Institute. Available at: http://seer.cancer.gov/csr/1975_2006 Accessed January 29, 2010.

10. National Cancer Institute. Survivorship. 2010. Available at: http://www.cancer.gov/search/results.aspx Accessed September 2, 2010.

11. Nelson JE, Meier DE, Oei EJ, et al. Self-reported symptom experience of critically ill cancer patients receiving intensive care. Crit Care Med. 2001;29:277-282.

12. Anderson KO, Mendoza TR, Valero V, et al. Minority cancer patients and their providers: pain management attitudes and practice. Cancer. 2000;88:1929-1938.

13. Caraceni A. Evaluation and assessment of cancer pain and cancer pain treatment. Acta Anaesthesiol Scand. 2001;45: 1067-1075.

14. Fortner BV, Okon TA, Portenoy RK. A survey of painrelated hospitalizations, emergency department visits, and physician office visits reported by cancer patients with and without history of breakthrough pain. J Pain. 2002;3:38-44.

15. Green CR, Montague L, Hart-Johnson T. Consistent and breakthrough pain in diverse advanced cancer patients: a longitudinal examination. J Pain Symptom Manage. 2009; 37:831-847.

16. Montague L, Green CR. Cancer and breakthrough pain's impact on a diverse population. Pain Med. 2009;10:549-561.

17. Anderson KO, Green CR, Payne R. Racial and ethnic disparities in pain: causes and consequences of unequal care. J Pain. 2009;10:1187-1204.

18. Green CR, Anderson KO, Baker TA, et al. The unequal burden of pain: confronting racial and ethnic disparities in pain. Pain Med. 2003;4:277-294.

19. Sondik EJ, Lucas JW, Madans JH, Smith SS. Race/ethnicity and the 2000 census: implications for public health. Am J Public Health. 2000;90:1709-1713.

20. Ibrahim SA, Burant CJ, Siminoff LA, Stoller EP, Kwoh CK. Self-Assessed Global Quality of Life: a comparison between African-American and white older patients with arthritis. J Clin Epidemiol. 2002;55:512-517. 
21. Glanz K, Croyle RT, Chollette VY, Pinn VW. Cancerrelated health disparities in women. Am J Public Health. 2003;93:292-298.

22. Bach PB, Schrag D, Brawley OW, Galaznik A, Yakren S, Begg CB. Survival of blacks and whites after a cancer diagnosis. JAMA. 2002;287:2106-2113.

23. Fuentes M, Hart-Johnson T, Green CR. The association among neighborhood socioeconomic status, race and chronic pain in black and white older adults. J Natl Med Assoc. 2007;99:1160-1169.

24. Satcher D. The unequal burden of cancer. Cancer. 2001;91: 205-207.

25. Green CR, Baker TA, Ndao-Brumblay SK. Patient attitudes regarding healthcare utilization and referral: a descriptive comparison in African- and Caucasian Americans with chronic pain. I Natl Med Assoc. 2004;96:31-42.

26. Institute of Medicine. Unequal Treatment: Confronting Racial and Ethnic Disparities in Health Care. Washington, DC: National Academies Press; 2002.

27. Bernabei R, Gambassi G, Lapane K, et al. Management of pain in elderly patients with cancer. SAGE study group. Systematic assessment of geriatric drug use via epidemiology. JAMA. 1998;279:1877-1882.

28. Green CR, Ndao-Brumblay SK, West B, Washington T. Differences in prescription opioid analgesic availability: comparing minority and white pharmacies across Michigan. J Pain. 2005;6:689-699.

29. Morrison RS, Wallenstein S, Natale DK, Senzel RS, Huang LL. "We don't carry that"-failure of pharmacies in predominantly nonwhite neighborhoods to stock opioid analgesics. N Engl J Med. 2000;342:1023-1026.

30. Dillman DA. Mail and Telephone Surveys: The Total Design Method. New York, NY: Wiley-Interscience; 1978.

31. McDowell I, Newell C. Measuring Health: A Guide to Rating Scales and Questionnaires. 2nd ed. New York, NY: Oxford University Press; 1996.

32. Schroevers MJ, Sanderman R, van Sonderen E, Ranchor AV. The evaluation of the Center for Epidemiologic Studies Depression (CES-D) scale: depressed and positive affect in cancer patients and healthy reference subjects. Qual Life Res. 2000;9:1015-1029.

33. James SA, Hartnett SA, Kalsbeek WD. John Henryism and blood pressure differences among black men. J Behav Med. 1983;6:259-278.
34. Gunnarsdottir S, Donovan HS, Serlin RC, Voge C, Ward S. Patient-related barriers to pain management: the Barriers Questionnaire II (BQ-II). Pain. 2002;99:385-396.

35. Tait RC, Chibnall JT, Krause S. The Pain Disability Index: psychometric properties. Pain. 1990;40:171-182.

36. Aaronson NK, Ahmedzai S, Bergman B, et al. The European Organization for Research and Treatment of Cancer QLQC30: a quality-of-life instrument for use in international clinical trials in oncology. J Natl Cancer Inst. 1993;85:365-376.

37. Institute of Medicine. The Unequal Burden of Cancer: An Assessment of NIH Research and Programs for Ethnic Minorities and the Medically Underserved. Washington, DC: National Academy Press; 1999.

38. Jensen MP, Chang HY, Lai YH, Syrjala KL, Fann JR, Gralow JR. Pain in long-term breast cancer survivors: frequency, severity, and impact. Pain Med. 2010;11:1099-1106.

39. Deimling GT, Sterns S, Bowman KF, Kahana B. The health of older-adult, long-term cancer survivors. Cancer Nurs. 2005;28:415-424.

40. Griggs JJ, Culakova E, Sorbero ME, et al. Social and racial differences in selection of breast cancer adjuvant chemotherapy regimens. J Clin Oncol. 2007;25:2522-2527.

41. Travis SS, Bernard M, Dixon S, McAuley WJ, Loving G, McClanahan L. Obstacles to palliation and end-of-life care in a long-term care facility. Gerontologist. 2002;42:342-349.

42. Green CR, Ndao-Brumblay SK, Nagrant AM, Baker TA, Rothman E. Race, age, and gender influences among clusters of African American and white patients with chronic pain. J Pain. 2004;5:171-182.

43. Green CR, Baker TA, Sato Y, Washington TL, Smith EM. Race and chronic pain: a comparative study of young black and white Americans presenting for management. J Pain. 2003;4:176-183.

44. Cleeland CS. Undertreatment of cancer pain in elderly patients. JAMA. 1998;279:1914-1915.

45. Weisse CS, Sorum PC, Dominguez RE. The influence of gender and race on physicians' pain management decisions. J Pain. 2003;4:505-510.

46. Abraham KG, Maitland A, and Bianchi SM. Nonresponse in the American time use survey. Public Opin Q. 2006;70: 676-703.

47. Komenaka IK, Martinez ME, Pennington RE Jr, et al. Race and ethnicity and breast cancer outcomes in an underinsured population. J Natl Cancer Inst. 2010;102:1178-1187. 